Marie PERRIN, «Victor Hugo, un classique romantique? », @nalyses, automne 2006

\title{
Marie PERRIN
}

\section{Victor Hugo, un classique romantique?}

Dans ce premier ouvrage à la fois réfléchi et fouillé, la publication d'un DEA soutenu en 2002 à Paris-IV Sorbonne, Bernard Le Drezen entend «poser les prolégomènes d'une étude plus vaste de l'éloquence politique au milieu du [XIX $]$ siècle » (104) en s'appuyant sur la figure de Hugo, auteur emblématique dont l'activité parlementaire est à cette époque particulièrement significative. Il se place, ce faisant, dans la tendance actuelle d'études qui soulignent non la « rupture » associée de manière traditionnelle au romantisme, mais les liens forts que le mouvement et ses représentants entretiennent avec les conceptions humanistes et «classiques » : l'éloquence est ainsi un morceau de choix pour qui se place sur ce terrain.

L'étude se donne pour objectif principal la mise en valeur de l'articulation du politique et du littéraire à partir du premier tome d'Actes et Paroles (1841-1851), au cours d'une période capitale pour l'évolution politique, littéraire et personnelle de Hugo. Bernard Le Drezen se recentre plus particulièrement, dans son quatrième et dernier chapitre, sur les débats qui entourent l'Affaire de Rome, dans la mesure où le discours que Hugo prononça à cette occasion (octobre 1849) constituerait le premier perçu à l'époque comme étant d' "opposition », alors que Guy Rosa situe plutôt ce virage au moment du discours sur la liberté d'enseignement (15 janvier 1850). Mais polémiquer sur les dates ne rentre pas dans le projet de l'auteur, puisqu'une de ses hypothèses de départ est qu'il n'y a pas chez Hugo " de coupure radicale [...] entre l'orléanisme et le républicanisme » (20) en raison d'une continuité dans la réflexion sur le langage et la rhétorique, qui entretiennent d'étroites relations avec le politique.

Au-delà de simples "prolégomènes ", le livre est déjà en lui-même particulièrement riche et solide, nourri par un support historique et bibliographique de qualité, bien que l'analyse proprement dite 
n'excède pas en elle-même 155 pages, la deuxième partie — soit un peu moins de la moitié — étant composée d'une bibliographie détaillée et d'annexes. Ces dernières, constituées de discours parlementaires publiés dans leur intégralité, permettent d'éclairantes comparaisons: comparaison entre un discours de Hugo paru au Moniteur et sa version réécrite dans Actes et Paroles, mais aussi comparaison avec des orateurs parlementaires contemporains tels le brillant Montalembert, « orateur total» (136), le ministre Tocqueville, «orateur démocratique» (126), ou le beaucoup plus obscur républicain Mathieu de la Drôme. Le choix d'une date resserrée, du 18 au 20 octobre 1849, autour d'une question précise — l'affaire romaine et la légitimité du motu proprio ainsi que celle de l'éphémère République romaine que la France venait de combattre en rétablissant le pouvoir temporel du pape - permet de comprendre comment les discours des orateurs se répondent et s'enchaînent, la tribune n'étant nullement «autotélique ». Plus proche du théâtre et de la chaire, la tribune implique pour Hugo des conceptions langagières qui permettent néanmoins de mieux comprendre l'ensemble de son œuvre, y compris romanesque.

Il faut passer outre certains détails trompeurs qui masquent presque plaisamment la très grande qualité du propos : si des titres de la table des matières semblent par exemple peu évocateurs du sujet qu'ils traitent, tels qu' Assimilation, rejet et approfondissement des problématiques » ou «Enjeux et ressaisissement des problématiques globales », les sous-parties rétablissent immédiatement la clarté et la précision du développement. Car telles sont bien deux qualités majeures de Bernard Le Drezen : la limpidité et la rigueur au service de remarquables qualités de synthèse.

La réflexion s'articule en quatre chapitres. Le premier, qui traite des rapports conflictuels entre littérature et politique au XIX ${ }^{\mathrm{e}}$ siècle, est une justification de l'étude littéraire des discours parlementaires, dans le prolongement de l'introduction. Le traitement littéraire des œuvres politiques de Hugo ne va en effet pas de soi et constitue, comme le rappelle l'auteur, le parent pauvre des études hugoliennes, alors même que Hugo et ses contemporains jugeaient, pour le meilleur ou pour le 
pire, les deux domaines indissociables. Une conception de plus en plus restrictive du champ littéraire et la professionnalisation d'une politique toujours plus technique rendent compte de ce phénomène, alors que pour Hugo, «la prise de parole du grand orateur n'est pas séparée des autres activités humaines» (32). Hugo le romantique est ainsi rapproché de l'idéal classique et humaniste, et le "mage » romantique voit converger dans sa personne les fonctions antiques de l'orator « vir bonus dicendi peritus » et celles du pasteur. L'éloquence révolutionnaire elle-même, loin d'être un renouvellement, est nourrie de références antiques.

Bernard Le Drezen s'attache ensuite à définir minutieusement ses outils méthodologiques face à des textes «troués », comme au théâtre, à «mi-chemin entre l'écrit et le dit» (36) et dont l'actio est à jamais perdue pour nous. À cela s'ajoutent des difficultés d'interprétation liées à une transcription des discours souvent fautive ou lacunaire dans Le Moniteur universel, ainsi que des difficultés de compréhension littérale de textes dont l'actualité et le contexte précis nous échappent souvent. Les premiers outils qui s'offrent à l'analyse de pareils discours politiques sont inévitablement les références aux modèles rhétoriques antiques. Mais pour ne pas tomber dans le travers qui consiste à appliquer leurs catégories de manière systématique à n'importe quelle prise de parole, au risque d'en détruire la cohérence et la vie, Bernard Le Drezen rappelle la nécessité de s'appuyer sur la conscience «métarhétorique» (39) marquée qui existe chez Hugo. Quant aux plus intéressantes approches contemporaines, elles permettent d'appréhender le discours politique comme champ de force et de confrontations qui visent à emporter l'adhésion en proposant une interprétation du monde.

Le second chapitre est un rappel de la position ambiguë de Hugo face à la rhétorique : le rejet romantique provocateur masque en effet une très grande familiarité avec les grands orateurs - Cicéron ou Quintilien - dont l'écrivain maîtrise et exploite les codes de manière virtuose. Ce qui est refusé par Hugo, c'est l'artifice rhétorique, qui crée une langue stérile aux dépens d'une langue simple et directe, enracinée dans la sensibilité. Bien que cela soit moins explicite, les modèles 
hugoliens, dans leur diversité même, soulignent cette ambiguïté, l'orateur révolutionnaire Mirabeau côtoyant l'orateur sacré, et le conservateur Chateaubriand ne devant pas faire oublier le républicain Lamartine. Mirabeau ainsi «invente l'éloquence moderne tout en retrouvant la fonction civique traditionnelle de l'orators (57). La glorification de l'éloquence révolutionnaire qu'il incarne est déjà romantique parce qu'il a vécu la souffrance dont il témoigne, mais elle repose également sur le postulat traditionnel et classique qu'une parole fondatrice est «fondée et confirmée par la valeur morale de celui qui l'exerce » (J. Starobinski, cité p. 56). Quant à la chaire, seul endroit où pouvait se développer une parole publique avant la Révolution, elle est liée à l'écoute d'une voix intérieure avant toute prise de parole, « un approfondissement se traduisant par un rayonnement extérieur» (61). C'est elle, dont la fonction première est de déclencher la méditation par le biais de l'émotion, qui inspire la rhétorique du cœur qui est celle de Hugo.

La conception du verbe hugolien, à la fois parole indissociable de l'idée - «transparence du langage à la vérité » (67) et «fantasme de la parole-acte» (69) qui inclut la violence —, clôt le chapitre. La conciliation des catégories de l'idée et de la force est permise au sein de la beauté, valeur suprême du discours dans la mesure où le beau possède une force persuasive certaine. Mais l'auteur ne différencie pas toujours nettement le beau du sublime, le sublime rhétorique du sublime esthétique (pour reprendre une distinction de G. Molinié). Il rappelle que le «beau hugolien inclut la surprise, l'irrégularité, et une certaine quantité de violence, ce qui le rapproche des définitions habituelles du sublime » (71), mais son objet d'étude l'amène, semble$\mathrm{t}$-il, à privilégier une conception du sublime comme « association de la grandeur et de la beauté » (73), comme énergie, force et véhémence, dans la tradition rhétorique de la notion. Les discours politiques hugoliens témoignent en effet avant tout de la clarté, de la noblesse et de la beauté liées aux exigences de hautes valeurs morales et intellectuelles, qui n'incluent guère «d'horreurs délicieuses» ou autres oxymores liés au sublime esthétique. Quant au grotesque, Bernard Le Drezen remarque qu'il est absent du discours pour mieux se trouver 
dans l'auditoire, et singulièrement du côté du parti de l'Ordre, l'orateur assumant la part de sublime.

Le troisième chapitre est, avec le dernier, un des plus remarquables et s'articule sur la célèbre opposition entre la raison et le cœur, l'ethos et le pathos, au sein même d'une «anthropologie hugolienne» qui débouche sur une étude de l'orateur démocratique.

L'éloquence de Hugo revendique un monopole du cœur qui fait de la persuasion par le sentiment une constante dans ses discours, voire dans l'ensemble de son œuvre (79). Bernard Le Drezen parle plus particulièrement d'«anthropologie hugolienne» dans la correspondance qui s'établit entre le cœur et l'esprit, entre l'âme et le sentiment, notamment lorsque l'écrivain affirme : «La hauteur des sentiments est en raison directe de la profondeur de l'intelligence » (Massin, t. VII, p. 64, cité p. 79). Puisque l'émotion a une valeur heuristique qui permet l'accès à la vérité, une des conséquences majeures sur le plan du discours est l'importance du recours aux figures, qui se substituent souvent au raisonnement et ont tendance «à faire argument à elle[s] seule[s]» (81), comme la métaphore filée.

Au monopole du cœur il faut ajouter la réflexion sur le bon usage des passions en politique, à la suite de Mme de Staël. Un contrôle strict des passions serait ainsi l'apanage du discours hugolien : il s'agit en effet avant tout, sous la monarchie de Juillet et la Deuxième République, de rétablir l'ordre et l'harmonie dans la cité et de condamner un certain type de passions, «celles qui isolent les individus, promeuvent l'intérêt individuel et la jouissance matérielle comme valeur suprême » (84). La condamnation des Lumières et du voltairianisme, au début de sa carrière, s'inscrit dans une hantise de déliaison de la communauté par la raison et par l'espace public qu'elle présuppose, qui persistera tout au long de sa vie. La crise de la représentativité qui s'ouvre pendant la monarchie de Juillet nécessite le rappel d'une «esthétique du pouvoir bon et juste » (87), représentée par les temps bénis de la Constituante.

La dénonciation du matérialisme est une constante qui ne s'accompagne pourtant jamais, chez Hugo, d'une dévaluation de la 
parole. On parvient ainsi au paradoxe formulé de la manière suivante : «la pratique hugolienne de la parole présuppose un espace public, même si Hugo utilise cet espace pour y promouvoir des valeurs qui sont en contradiction avec les modalités de leur lieu d'exercice. En effet, l'espace public est lié à l'usage libre de la raison. Mais cet usage de la raison est nécessairement désacralisant et destructeur des liens sociaux... Situation paradoxale qui contient les germes de son échec. D'où l'intérêt pour Hugo de convaincre que l'intelligence et le cœur ne sont qu'une seule et même chose, et que les progrès de la raison n’impliquent pas le triomphe de l'individualisme » (88).

Dans le passage de Hugo de l'orléanisme à un certain socialisme, le changement se situe dans le rapport aux passions : jusqu'à « un peu avant $1850 »(88)$, il s'agit avant tout de participer à leur apaisement, bien que l'éloquence hugolienne les accepte au sein du discours. Mais ce que découvre Hugo lors de sa «conversion », c'est que «l'on peut faire un bon usage des passions, et que cette conciliation des passions, leur utilisation heureuse, coïncide avec l'idéal républicain » (89), qui grandit l'homme. Le mot d'ordre de la littérature et de l'éloquence devient le suivant: "par l'intermédiaire d'un usage raisonnable des passions positives (l'amour, la générosité, la bonté), créer du lien social» (89), en réaction aux ennemis de la République, qui sont animés par la haine et l'esprit de revanche. On peut néanmoins ajouter que cette conception n'est que transitoire: elle s'infléchira encore pendant l'exil, la situation politique étant tout autre. Colère et indignation sont alors valorisées au détriment de l'apaisement, associé à la passivité, voire à la lâcheté. Et le but suprême de l'écrivain n'apparaitra pas uniquement sous l'idéal classique de «dévouer sa pensée au développement continu de la sociabilité humaine » (Massin, t. VI, p. 161, cité p. 89), mais fera appel au modèle révolutionnaire.

La question qui se pose dans un second temps est de savoir «dans quelle mesure la conception hugolienne de la représentation et la conviction républicaine, assez tardive, sont concurrentes ou compatibles » (90). Les solutions violentes répugnent à Hugo à la fin de la monarchie de Juillet: jusqu'à l'exil, il n'adopte jamais les positions les plus radicales de la gauche. Mais de manière générale, son 
positionnement idéologique depuis les années 1830 n'est pas en contradiction fondamentale avec celui de l'exil: en matière économique par exemple, il défendra toujours farouchement la nécessaire liberté de la production et des échanges, élément fondamental du credo libéral. Son ralliement aux idées de gauche n'est que partiel. Une pareille continuité se lit également dans l'idée de gouvernement direct, en germe dès les années 1830, passée sous silence au cours de la Deuxième République, qui privilégie le système représentatif, incarnation de la volonté populaire au détriment de la démocratie directe, et qui ressurgit dans Napoléon le Petit, une fois que l'Assemblée législative semble aller à l'encontre des intérêts du peuple.

La conviction républicaine de la représentation contractuelle n'intervient qu'une fois évacuée la tentation bonapartiste de la représentation du pouvoir dans un seul homme - ce qui était une manière de résoudre, par l'individualisation du pouvoir, les problèmes posés par le gouvernement représentatif, rarement représentatif et n'aboutissant jamais à l'unanimité. "Le schéma du peuple devenu souverain, Victor Hugo étant son prophète, n'est pas non plus tout à fait indemne de cette tentation » (96). Quoi qu'il en soit, la représentation populaire est par la suite pensée, en réaction, comme identique à la souveraineté populaire en exercice. La République, «commencement du fait moral » (Massin, t. VII, p. 282, cité p. 98), met au jour les liens sociaux et se révèle incompatible avec l'idée de l'homme providentiel : l'idéal démocratique ne peut reposer sur l'adoration d'un seul homme, dans la mesure où la contradiction « fait partie intégrante du travail de l'idée et de la découverte du vrai » (98). L'intégration de cette contradiction dans le discours et l'œuvre en général devient ainsi une des caractéristiques de l'esthétique hugolienne. En termes rhétoriques, on parlera de rétorsion.

On aboutit ainsi à la fonction du poète et de l'orateur comme affirmation du lien entre les hommes et à la définition de la littérature comme lieu de partage. La conscience et la responsabilité de l'orateur ont à voir avec le divin et son cœur fait corps avec celui du peuple qu'il représente: il s'agit là encore d'une constante de l'éloquence hugolienne qui ne date pas de sa conversion démocratique, l'appel au 
peuple par-dessus l'Assemblée nationale étant par ailleurs un lieu commun de l'éloquence, particulièrement républicaine. Cette position de parole amène Hugo, par le biais de ses discours, à redéfinir la littérature comme lieu commun, « au sens où elle est le réceptacle et la caisse de résonance de préoccupations communes et universelles » (102). Ce qui est une manière élégante de rappeler le contenu réel des discours, où se manifestent «la volonté nette de jouer sur les lieux communs parce qu'ils sont essentiels, quoique le plus souvent méprisés » dans une optique de "pédagogie républicaine » (102). Cette volonté d'établir des valeurs communes afin d'assurer la cohésion de la nation permet à Hugo de retrouver les fonctions traditionnelles de l'éloquence politique.

Enfin, le quatrième chapitre, qui s'appuie sur les annexes, étudie la manière dont Hugo s'intègre aux luttes oratoires de ses contemporains, lors du débat portant sur le vote de crédits supplémentaires pour l'intervention française à Rome, du 18 au 20 octobre 1849. La date est fondamentale, on l'a dit, puisque Montalembert fixe à ce moment le basculement définitif de Hugo dans l'opposition. Quant à l'analyse minutieuse de ce débat parlementaire qui s’appuie sur des exemples précis (Hugo, Thiers, Tocqueville, Mathieu de la Drôme et Montalembert), elle est à la fois passionnante et éclairante. L'éloquence de Tocqueville, qui est «tout entière fondée sur le refus des passions»(126), est un excellent contrepoint à la rhétorique hugolienne. De même celle de Montalembert : sa position majoritaire à l'Assemblée lui permet d'user d'un humour et d'une ironie dont le caractère allusif procède des codes de la conversation mondaine, à la différence de l'éloquence démocratique fondée sur le rappel de valeurs et de souvenirs communs, en premier lieu celui de la Révolution française. Le discours du méconnu républicain Mathieu de la Drôme permet ainsi de comprendre combien les propres discours de Hugo manifestent « une solidarité totale avec le siècle qui leur a donné vie et les explique » (R. Badinter, préface, p. 8). La glorification de la tribune, instance suprême, et l'unité nécessaire des citoyens en République apparaissent ainsi comme des passages obligés pour tout orateur républicain. 
Plus profondément, le débat met en valeur les différentes idéologies du siècle qui suturent les discours, que ce soit la définition des positions respectives de l'Église et de l'État, la mise en doute de l'idéologie progressiste, «vraie foi de notre âge », pour Pierre Larousse (cité p. 143) ou l'interprétation de la notion de Liberté, associée pour les Républicains à la souveraineté du Peuple et à la Révolution, alors que Montalembert les dissocie : pour lui, les Républicains ont préféré « la révolution à la liberté » (146).

Hugo a été particulièrement raillé pour sa "politique des idées », mais Bernard Le Drezen montre, par sa riche étude, combien les idées importent dans un débat parlementaire, seules aptes à proposer « une vision de l'action collective et de l'avenir » (151). Cela est possible dans la mesure où Hugo a par ailleurs un regard extrêmement critique sur l'éloquence, dont il souhaite même à terme, de manière surprenante et originale, la disparition, lors de la résorption du conflit entre le droit et la loi dont elle est pour lui l'émanation : « ce sera le règne paisible de l'incontestable; on ne fera plus les lois, on les constatera; les lois seront des axiomes; on ne met pas aux voix deux et deux font quatre [...]» (cité p. 153). Peut-être faudrait-il néanmoins rappeler que cette utopie, qui reprend le discours de Cimourdain dans Quatrevingt-treize comme le rappelle Bernard Le Drezen - doit être impérativement nuancée par le refus que Gauvain lui oppose. Car cette profession de foi scientiste s'apparente à un fanatisme de la raison, synonyme de Terreur et non de Liberté, et signe la mort de toute parole, sinon de toute esthétique. Du moins si l'on entend par esthétique l'un des éléments essentiels de l'écriture hugolienne, l'accueil et la «mise en scène » — plus particulièrement à partir de l'exil — de toutes les voix divergentes qui la construisent, que ce soit pour montrer leur hétérogénéité dialogique ou bien au contraire leur fraternité contradictoire.

Référence: Bernard Le Drezen, Victor Hugo ou l'éloquence souveraine. Pratiques et théorie de la parole publique chez Victor Hugo, préface de Robert Badinter, L'Harmattan, coll. « Langue \& Parole », 2005, 271 p. 